\title{
Review: organisational change and patient directed strategies may increase adult immunisation and cancer screening
}

Stone EG, Morton SC, Hulscher ME, et al. Interventions that increase use of adult immunization and cancer screening

services: a meta-analysis. Ann Intern Med 2002;136:641-51.

\section{QUESTION: What is the effectiveness of interventions to increase adherence to guideline based adult immunisation and cancer screening?}

Source of funding: Centers for Medicare and Medicaid Services

For correspondence: Dr P G Shekelle, RAND, Santa Monica, CA, USA.

shekelle@rand.org cancer screening. Provider feedback may be least effective.

Studies were identified by searching (through February 1999) the Cochrane Effective Practice and Organization of Care Special Register (which includes searches of Medline [from 1966], EMBASE/Excerpta Medica [from 1980], HealthSTAR [from 1975], and the Cochrane Controlled Trials Register [from 1996]), previous systematic reviews, and the Health Care Quality Improvement Projects database.

\section{Study selection}

Studies were selected if they were controlled clinical trials that assessed interventions to increase the use of immunisations for influenza and pneumococcal pneumonia, and use of screening for colon, breast, and cervical cancer in adults.

\section{Data extraction}

Data were extracted on interventions (classified as reminder, provider feedback, education, financial incentive, legislative action, organisational change, or mass media campaign), study characteristics, and outcomes.

\section{Main results}

108 studies on immunisation (29 studies), mammography (33 studies), cervical cytology (27 studies), and colon cancer screening (19 studies) met the inclusion criteria. Of these, 95 were randomised controlled trials (RCTs) and 13 were controlled clinical trials. 81 studies had a usual care or control group and were eligible for metaregression analysis. Of these 81 studies, 70 were RCTs, of which 22 allocated at the provider, organisation, or community level. The effectiveness of different types of interventions for increasing the use of immunisation and screening services is shown in the table. Organisational changes, such as use of separate clinics aimed at screening and prevention services, use of a planned care visit for prevention, use of continuous quality improvement techniques, or designation of non-physician staff to do specific prevention activities, appeared most effective for increasing the use of immunisation and cancer screening. Patient directed financial incentives, reminders, and education also appeared effective. Provider feedback seemed to be least effective.

\section{Conclusions}

Healthcare organisational changes and patient directed strategies such as financial incentives, reminders, and education are likely to show positive effects on
Interventions for increasing the use of immunisations, mammography, cervical cytology, or colon cancer screening

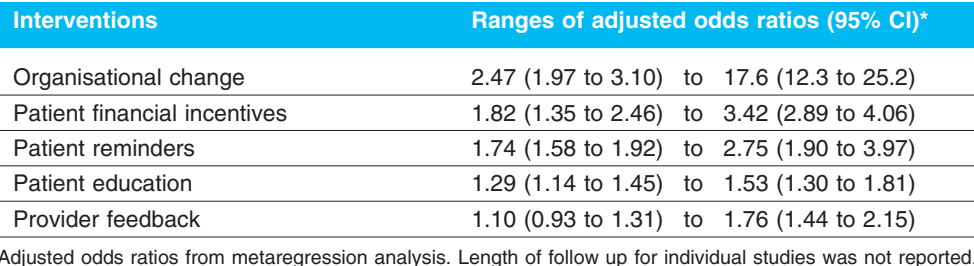

\section{COMMENTARY} health services emphasising primary and secondary prevention have increased markedly over the past few decades. ${ }^{1}$ Because many adults do not use immunisation and cancer screening services, research findings pointing to tangible ways to improve adult patients' adherence with these services are important.

The meta-analysis by Stone et al combines the strength of a well conceptualised theoretical framework with rigorous research methods. The framework considered the types of interventions tested, the targets of the interventions, and the features of the interventions designed to increase their potency. Other strengths of the review include a comprehensive literature search, careful study selection and data abstraction, and a multivariate modelling approach to data analysis. Unfortunately, the relative cost effectiveness of the interventions could not be examined because their costs and benefits were not reported in sufficient detail in the individual studies. able resources to provider feedback strategies because the review showed that these were the least effective strategies. The most effective intervention was organisational change; this finding suggests that efforts should focus on the establishment of separate clinics devoted to prevention and screening activities, use of planned care visits specifically for prevention, use of continuous quality improvement strategies, and designation of responsibilities to non-physician staff. The philosophical basis of nursing supports a focus on preventive services. In addition, many nurses possess the requisite clinical and administrative knowledge and skills to do these roles. In some settings, the use of protocols might be required, but in others where nurses have a wider scope of practice, independent practice will be possible.

Clinicians, health administrators, and health policy makers will benefit from these results. The findings are likely applicable to many settings, including primary care settings, physician offices, outpatient clinics, and public health agencies. When implementing 1 or more of these strategies, consideration should be given to examining their cost effectiveness.

Linda Hilts, RN, MFd Family Practice Nurse, Stonechurch Family Health Centre, Assistant Clinical Professor, School of Nursing McMaster University, Hamilton, Ontario, Canada

1 Hensrud DD. Clinical preventive medicine in primary care: background and practice: 1 . Rationale and current preventive practices. Mayo Clin Proc 2000;75:165-172.
Although the treatment of symptomatic disease and illness still dominates health care,

Many organisations may want to rethink the current strategy of allocating consider- 